\title{
Grupos de Ajuda Mútua para Cuidadores Informais de pessoas com demência: no sentido de um helping ethos comunitário
}

\author{
I 1 Óscar Ribeiro, ${ }^{2}$ Rosa Almeida, ${ }^{3}$ Cristina Barbosa, ${ }^{4}$ Natália Duarte, \\ ${ }^{5}$ Daniela Brandão I
}

Resumo: No âmbito das intervençôes comunitárias que são promovidas conjuntamente por entidades da área da saúde e do social, as intervençôes grupais desenvolvidas para cuidadores informais de pessoas com demência têm se instituído como um importante tópico de investigação e objeto de reflexão profissional. O presente estudo tem como objetivo expor o funcionamento de um conjunto de Grupos de Ajuda Mútua (GAM) enquanto respostas de continuidade no apoio a cuidadores informais de pessoas com demência que participam em Programas Psicoeducativos. Partindo de um Projeto de Intervenção Comunitária realizado na zona Norte de Portugal (Projeto Cuidar de Quem Cuida), o estudo descreve cinco GAMs ocorridos em municípios diferentes e implementados/dinamizados por agentes de desenvolvimento local. São apresentados os seus objetivos e principais características de funcionamento enquanto resposta de continuidade. Os resultados revelam a sua importância para os cuidadores, nomeadamente para a dinamização/ manutenção de redes de apoio social informal no âmbito das escassas respostas especificamente destinadas para esta população, bem como para o papel fundamental dos seus agentes dinamizadores (técnicos de saúde e/ou da ação social). Conclui-se pela relevância dos GAMs enquanto iniciativa comunitária de entreajuda e de suporte ativo e continuado para cuidadores informais de idosos com demência.

> Palavras-chave: cuidadores informais; demência; grupos de apoio; idosos.

\author{
1 Unidade de Investigação e \\ Formação sobre Adultos e \\ Idosos - Instituto de Ciências \\ Biomédicas Abel Salazar, \\ Universidade do Porto; Centro \\ de Investigação em Tecnologias \\ e Serviços de Saúde; Instituto \\ Superior de Serviço Social \\ do Porto. Porto, Portugal \\ (osribeiro@icbas.up.pt). \\ ${ }^{2}$ Fundación INTRAS. Valladolid, \\ Espanha (ralmeida@ides.es). \\ ${ }^{3}$ Universidade do Minho. Braga,
Portugal (cristinamobarbosa@) \\ gmail.com). \\ ${ }^{4}$ Unidade de Investigação e \\ Formação sobre Adultos e \\ Idosos - Instituto de Ciências \\ Biomédicas Abel Salazar, \\ Universidade do Porto. Porto, \\ Portugal (nduarte@unifai.eu). \\ ${ }^{5}$ Unidade de Investigação e \\ Formação sobre Adultos e \\ Idosos - Instituto de Ciências \\ Biomédicas Abel Salazar, \\ Universidade do Porto. Porto, \\ Portugal (daniela.brandao@ \\ unifai.eu).
}

Recebido em: 20/10/2016 Aprovado em: 30/05/2017 


\section{Introdução}

O número de casos de demência tem aumentado por todo o mundo concomitantemente com o crescimento da expectativa média de vida. Os seus sintomas e as suas consequências são negativos para os doentes, mas também para os familiares que assumem a prestação de cuidados (DUCHARME et al., 2014). Estes experienciam, com frequência, sobrecarga e estresse emocional por cuidarem de um ente querido, o que contribui também para consequências negativas na saúde psicossocial e para o aumento do risco de mortalidade (BRODATY et al., 2003).

Vários estudos têm demonstrado que intervenções desenvolvidas em grupo destinadas a cuidadores informais, como Grupos de Ajuda Mútua (GAM) ou Intervenções Psicoeducativas, nos quais é dada oportunidade aos membros para partilharem experiências e conhecimentos, são úteis no alívio da sobrecarga e na diminuição dos sintomas depressivos, bem como no favorecimento de comportamentos de saúde e de procura de apoio social, os quais colaboram para uma melhor qualidade de vida e satisfação com a tarefa de cuidar (CHIEN et al., 2011).

Os GAMs surgiram como os primeiros modelos de intervenção para cuidadores de pessoas com doença mental (JEREZ; LÓPEZ, 2008) e se definem como um "modelo social de saúde", que contempla aspetos emocionais, psicossociais e de saúde (SORIANO, 1998). Estes grupos são estruturas relativamente pequenas (6 a 15 elementos) constituídas por pessoas que partilham um problema e se reúnem para a resolução de dificuldades ou satisfação de uma necessidade (MAIA et al., 2002). Os GAMs fornecem apoio, reforço, informação e estratégias de coping para lidar de modo otimizado com o problema em causa. Além disso, decorrem, de um modo geral, sem uma estrutura de temas definida e são liderados pelos seus próprios membros, sendo autônomos ante a qualquer sistema externo (JEREZ; LÓPEZ, 2008).

Os GAMs se caracterizam, também, por facultarem um espaço de normalização terapêutica (Maia et al., 2002), que faculta significados aos seus participantes (NAVARRO, 2004), providenciando o sentido de identidade e de pertença (NAVARRO, 2005; GONÇALVES-PEREIRA; SAMPAIO, 2011). Centram-se no conceito terapêutico helping ethos, no qual o membro que ajuda mais o grupo é o que mais se ajuda a si próprio (FINN et al., 2009). Dados seu reconhecimento crescente em nível internacional e o incremento da sua popularidade, em 1986 
os GAMs orientados para os cuidadores informais foram legitimados, através

da Carta de Otawa, pela Organização Mundial da Saúde como um exemplo de participação comunitária (NAVARRO, 2005; SORIANO, 1998), ao motivar a reunião e partilha das necessidades de alguns subgrupos populacionais e ao fomentar o autocuidado e a ajuda mútua.

Um GAM para cuidadores é definido como um grupo de indivíduos que desempenham a função de prestadores informais de cuidados, habitualmente familiares da pessoa dependente, que se encontram regularmente em espaço propício para o estabelecimento de relaçóes de solidariedade (ARRUDA et al., 2008), troca de informação prática (e.g., sobre a doença, o cuidado, recursos da comunidade), assim como para dar e receber suporte emocional, facilitado por uma atitude comum de compreensão (PISTRANG et al., 2008). A sua finalidade última passa por combater e aprender a superar os problemas com os quais se deparam no decorrer da doença e do desenvolvimento do papel de cuidador (CHIEN, 2008), bem como promover um ajustamento emocional assente na perceção de que cada um dos participantes não está só na sua situação e pode, em conjunto, mobilizar as suas potencialidades - o chamado empowerment dos cuidadores (RAPPAPORT, 1993). Os GAMs constituem, assim, uma oportunidade para os cuidadores partilharem os seus sentimentos, problemas e conhecimentos com pares em situação semelhante (ALZHEIMER DISEASE INTERNATIONAL, 2000). Além disso, estes grupos permitem que o cuidador dedique maior atenção as suas próprias necessidades, concorrendo para um maior autocuidado (ALZHEIMER DISEASE INTERNATIONAL, 2000).

Enquanto que em países como os EUA e Reino Unido os GAMs para cuidadores são uma resposta com elevada expressividade numérica (CHIEN, 2008), possivelmente por possuírem uma vasta experiência no campo de associativismo e ajuda mútua (MAIA et al., 2002), nos países do sul da Europa, como Portugal, o despertar para este tipo de movimento e do exercício da cidadania das populações, em particular no campo da saúde, apresenta-se mais tardio (MAIA et al., 2002). São também escassas as reflexões sobre este tipo de intervenção enquanto resposta complementar/ de continuidade a outro tipo de medidas de apoio, como o acompanhamento individual ou a intervenção psicoeducativa (GUERRA et al., 2013). O presente artigo tem como objetivo expor as particularidades de funcionamento dos GAMs desenvolvidos no âmbito 
de um projeto de intervenção comunitária para cuidadores informais de pessoas com demência mais amplo - o Projeto Cuidar de Quem Cuida (CQC). São descritas as principais características destes grupos, como locais de realização, participantes, profissionais envolvidos, objetivos e atividades, e discutidas as configurações e modos de funcionamento observados nos GAMs. São também analisadas as potencialidades dos GAMs enquanto respostas de continuidade no apoio aos cuidadores após o término de um Programa Psicoeducativo.

\section{Enquadramento do Estudo: Projeto Cuidar de Quem Cuida}

O Projeto CQC teve a duração de 4 anos (2009-2013) e foi desenvolvido em Portugal na Região de Entre o Douro e Vouga (EDV), a qual é constituída por cinco conselhos pertencentes a uma sub-região da Região Norte e da Área Metropolitana do Porto (NUTSIII). Em 2008, a população total da Região EVD era de 277.020 habitantes (INSTITUTO NACIONAL DE ESTATÍSTICA, 2008). Trata-se de um projeto que teve como objetivo central promover respostas de apoio especializado aos cuidadores informais de idosos com demência da área geográfica indicada, e contemplou várias linhas de ação, das quais se destaca a implementação de 29 Programas de Intervenção Psicoeducativa que abrangeram 288 cuidadores informais. Estes Programas ambicionavam: i) ajudar o cuidador na compreensão da doença, ii) potenciar a qualidade dos cuidados prestados; iii) promover a diminuição da sobrecarga e do stresse decorrente do cuidar; e iv) promover redes de apoio social entre os cuidadores participantes (RIBEIRO et al., 2013). Cada Programa Psicoeducativo do CQC consistiu em dez sessões, com periodicidade semanal e duração de duas horas por sessão, perfazendo um total de 20 horas.

\section{Criação de respostas de continuidade após término do projeto}

$\mathrm{Na}$ sequência dos primeiros Grupos Psicoeducativos implementados em 2010, e considerando-se a necessidade de dar continuidade aos encontros entre os cuidadores informais abrangidos pelo projeto CQC, foram constituídos GAMs em cada um dos cinco municípios. Tendo os Grupos Psicoeducativos uma periodicidade e duração definidas, existiu a necessidade, identificada quer por técnicos, quer por cuidadores, de prolongar o apoio recebido (RIBEIRO et al., 2013). Os cuidadores participantes no Programa Psicoeducativo expressaram 
vontade de manter a pertença ao grupo no qual se encontravam inseridos e, nesse sentido, foi estudada a possibilidade da criação dos GAMs. Estes se assumiam, por um lado, como uma continuidade ao apoio prestado e às relações sociais de solidariedade estabelecidas interpares, e por outro, como um meio de promover independência e autonomia dos cuidadores ante a uma intervenção estruturada, como a promovida pelos Grupos Psicoeducativos, necessariamente dinamizadas por técnicos. Para a criação dos GAMs contribuiu, igualmente, a ausência de respostas especializadas para os cuidadores informais de pessoas com demência na área geográfica em causa. Mais que as necessidades técnicas, foi o potencial dos GAMs para ajudar o cuidador a compreender o seu familiar e a natureza do seu comportamento, incitar a reordenação da dinâmica familiar e a obtenção de recursos externos que propiciam a manutenção do cuidado no domicílio, assim como o sentimento de autocapacitação e estabilidade emocional, que justificou a formação dos grupos (GÓMEZ GÓMEZ, 2007).

De um modo geral, os GAMs se caracterizam por serem grupos cuja planificação, periodicidade de encontro e dinâmica de funcionamento distam significativamente dos Grupos Psicoeducativos. Uma vez que se tratava de uma metodologia para a qual os profissionais envolvidos no CQC não estavam familiarizados, foi desenvolvido um workshop com esta temática, dinamizado por um profissional da área da psicologia, e que contou com a presença de dez profissionais do projeto CQC. Na sequência desta formação foram desenvolvidos cinco GAMs, que, enquanto resposta de continuidade aos Grupos Psicoeducativos, acomodavam critérios de adequação às especificidades de cada município. Assim, os GAMs foram constituídos pelos cuidadores informais que se beneficiaram de intervenção psicoeducativa e que apresentaram vontade de integrar esta resposta (critério de inclusão). Cada GAM contou com a colaboração de um profissional, habitualmente um dos técnicos envolvidos na implementação dos Grupos Psicoeducativos.

\section{Metodologia}

Para a análise das características dos GAMs constituídos no âmbito do projeto CQC, foi considerada uma abordagem qualitativa (FLICK, 2005), assente na análise de conteúdo das informações recolhidas através de dois dispositivos de informação complementares, designadamente: a observação não participante 
da dinâmica de cada grupo e uma entrevista semiestruturada ao profissional facilitador/ dinamizador. Esta recolha de dados foi realizada por uma equipe de dois investigadores, coautores deste estudo, que estiveram presentes em pelo menos três sessões de cada GAM. Os dados recolhidos mediante observação (e.g., local da reunião, número de participantes e seu envolvimento na reunião, atividades realizadas e sua dinamização) foram registrados em diários de campo individuais e objeto de análise uma vez coligidos. A informação obtida foi depois apresentada ao dinamizador do grupo em causa que, através de uma entrevista semiestruturada, providenciou informação complementar sobre o funcionamento habitual do GAM (e.g., objetivos, dinâmica de funcionamento das sessões, perfil de participantes, eventuais particularidades do grupo, apreciação geral do seu funcionamento e benefícios percebidos para os cuidadores). As autorizaçôes relativas à presença dos investigadores para a recolha de dados foram solicitadas aos cuidadores e profissionais participantes (consentimento informado), salvaguardando-se o anonimato da informação recolhida. O projeto Cuidar de Quem Cuida teve aprovação da Comissão de Ética da Administração Regional de Saúde do Norte (Parecer no 53/2010).

\section{Resultados}

Os cinco GAMs desenvolvidos exibem uma periodicidade mensal, com reuniōes de duração aproximada de duas horas, em horário determinado pelos elementos participantes. Apesar destas características em comum, os GAMs apresentam singularidades, as quais refletem diferenças socioculturais inerentes a cada um dos municípios. As principais características dos GAMs encontram-se sistematizadas na Tabela 1. 


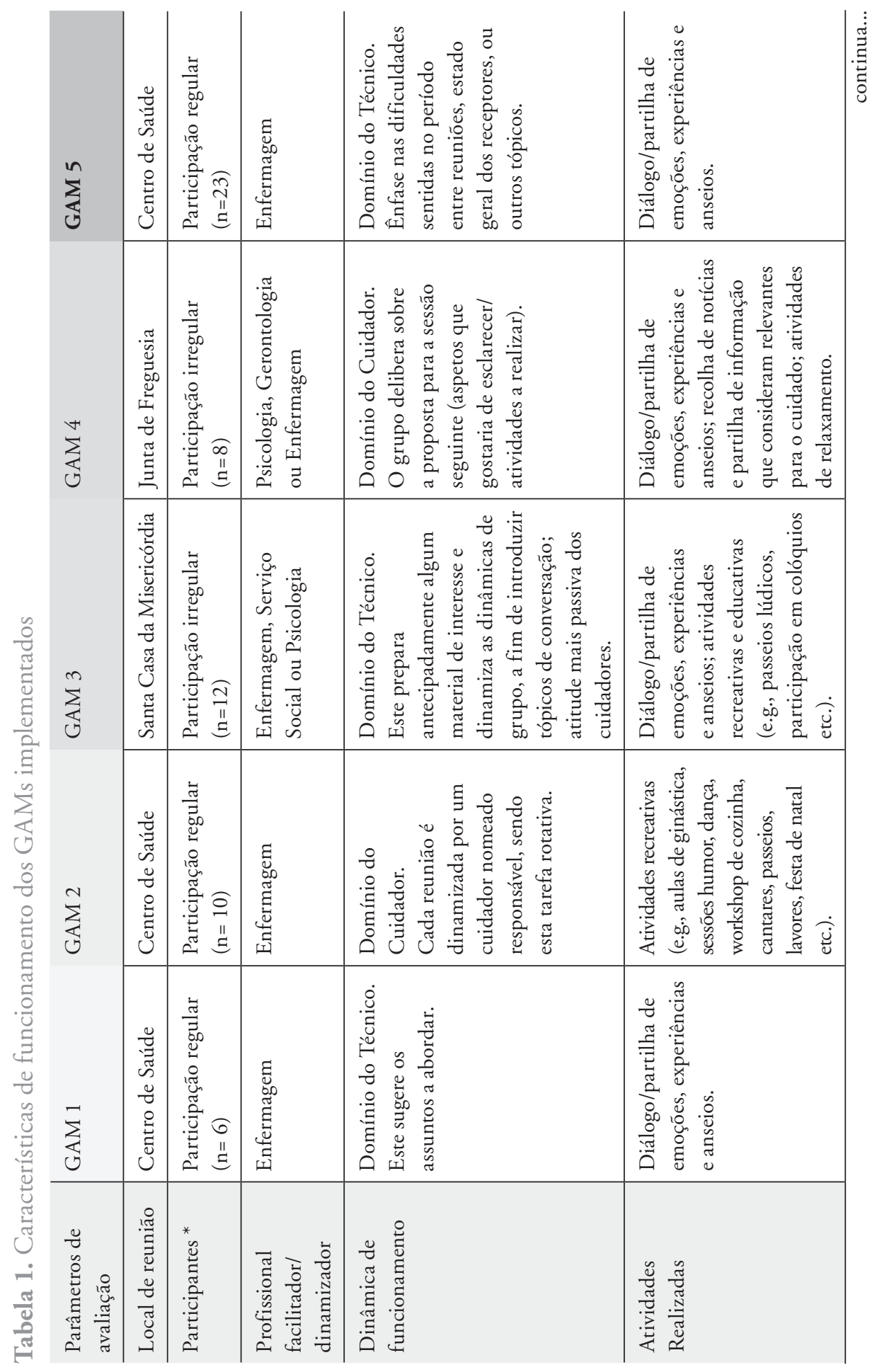


404

\begin{tabular}{|c|c|c|}
\hline$\sum_{0}^{n}$ & 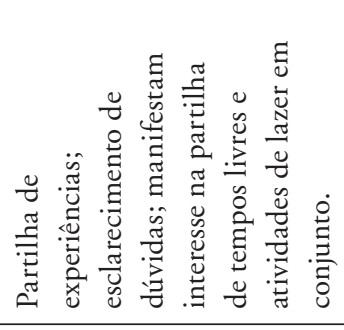 & 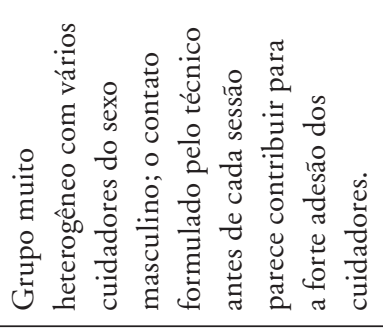 \\
\hline$\sum_{\circlearrowleft}^{+}$ & 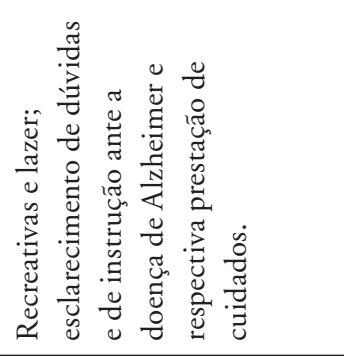 & 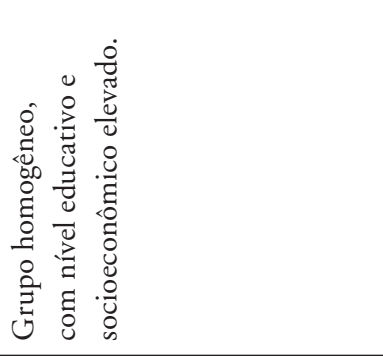 \\
\hline$\sum_{0}^{\infty}$ & 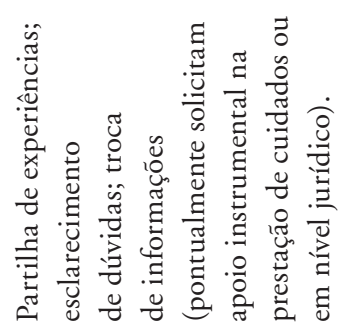 & 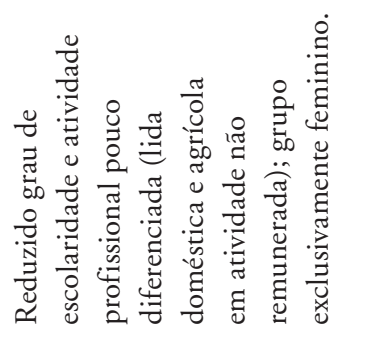 \\
\hline 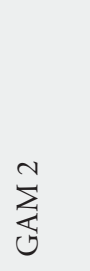 & 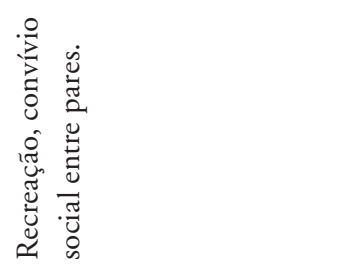 & 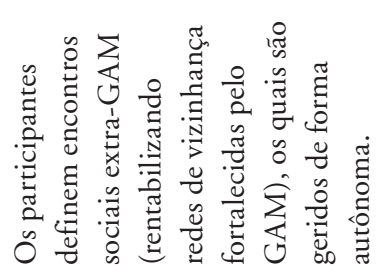 \\
\hline$\underset{\mho}{\Xi}$ & 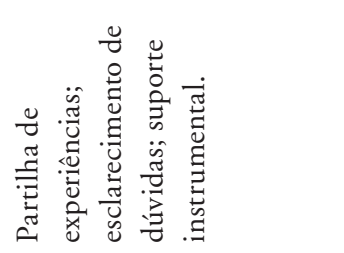 & \\
\hline 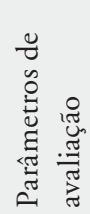 & 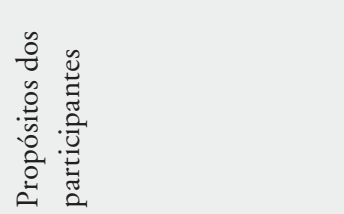 & 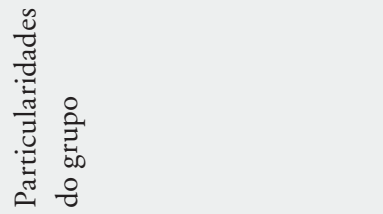 \\
\hline
\end{tabular}


Os locais de realização dos GAMs agruparam-se em Estabelecimentos de Saúde ( $\mathrm{n}=3)$, Equipamentos Sociais $(\mathrm{n}=1)$ e Juntas de Freguesia $(\mathrm{n}=1)^{1}$. O número de participantes presentes em cada grupo foi de 6 a 23 cuidadores; no entanto, a assiduidade dos integrantes comportou-se de forma distinta entre os grupos, sendo que nos GAMs 3 e 4 a participação dos cuidadores foi mais irregular que nos grupos restantes. Analisando as principais características sociodemográficas dos participantes, é também possível verificar uma elevada heterogeneidade entre os grupos. No GAM 2, participaram, em exclusivo, cuidadores do sexo feminino, contrapondo à existência de vários cuidadores do sexo masculino no GAM 5. De igual modo, o nível de escolaridade e o nível socioeconômico foram também características diferenciadoras entre os membros dos grupos.

No que se refere aos profissionais facilitadores/ dinamizadores, foram, na sua maioria, da área da Enfermagem. Os Grupos 3 e 4 apresentaram o suporte de profissionais da área da Psicologia, do Serviço Social e da Gerontologia. Observouse que os GAMs 1, 3 e 5 assentaram sobre um funcionamento protagonizado pelo profissional facilitador (que nestas condições toma também a função de dinamizador), e que nos GAMs 2 e 4 o domínio foi dos cuidadores, mas que apresentaram, ainda assim, grande heterogeneidade ao nível do formato participativo. Os profissionais implicados em cada grupo assumiram um papel relevante no apoio aos GAMs, desempenhando uma importante função ao moderar/equilibrar a participação dos cuidadores (e.g., evitando conversas paralelas e/ou a tentativa de monopólio da reunião por alguns participantes), ao facilitar a entrada de novos membros e estimular a sua participação, nortear a informação partilhada no grupo para que esta se adapte à condição individual de cada elemento e ao fomentar a participação ativa, evitando, dessa forma, a dependência do grupo quanto à presença do profissional, situação que se verificou em alguns GAMs.

Apesar de a maioria dos GAMs se centrar na partilha de experiências (i.e., estado atual da doença, situação familiar, desgaste sentido com a prestação de cuidados), informaçôes de interesse comum (e.g., recursos sociais na comunidade) e estratégias para lidar com problemas decorrentes da condição clínica dos receptores de cuidados (e.g., alterações de comportamento), e se concentrar igualmente na autocapacitação e encorajamento, o GAM 2 se distinguiu dos restantes e do próprio conceito de GAM para cuidadores expresso habitualmente na literatura. Este Grupo não procura momentos de partilha de experiências e 
suporte emocional, mas sim momentos aprazíveis de recreação, com a manutenção dos laços de amizade que fomentem o convívio extra-GAM e diálogos distantes da realidade quotidiana do cuidar.

Enquanto alguns GAMs evidenciaram maior preocupação com a prestação de apoio entre pares relativa à função de cuidador que executam (e.g., o apoio prático) (GAMs 1, 3, 4 e 5), outros dedicaram também os seus esforços à monitorização e análise crítica aos Serviços Sociais e de Saúde Mental que recebem ou que são disponibilizados na comunidade (GAM 4), assim como à obtenção de informação relevante sobre os avanços científicos mais recentes relativos à demência de Alzheimer (GAM 3).

\section{Discussão}

O desenvolvimento dos GAMs após o término dos Grupos Psicoeducativos do Projeto CQC surgiu de modo espontâneo e se baseou na necessidade de dar continuidade ao apoio prestado pelos técnicos e de suprir a "percepção de abandono" verbalizada pelos cuidadores na última sessão do Programa. O presente estudo pretendeu analisar as configurações e dinâmicas de funcionamento observadas nos GAMs desenvolvidos para estes cuidadores e expor as potencialidades desta resposta de entreajuda promovida em contexto comunitário.

Em primeiro lugar, importa destacar que, embora cada GAM apresente especificidades sobremaneira determinadas pelo contexto cultural em que se desenvolveu, a maior parte dos grupos exibiu a mesma periodicidade e duração das sessões e revelou a importância da manutenção das relações sociais interpares estabelecidas no seio dos grupos psicoeducativos. De fato, na observação não participante realizada nas sessões de cada um dos grupos, destacou-se a evidente interação entre os participantes, a qual denotava a existência de relações interpessoais sólidas e altamente valorizadas. Operacionalizando um "modelo social de saúde" (SORIANO, 1998) que contempla aspectos emocionais, psicossociais e de saúde traduzíveis num sentido de pertença a uma rede sólida e securizante, a maioria dos GAMs desenvolvidos centra a sua ação na partilha de experiências, de informações úteis e de estratégias para lidar com o problema, as quais têm como fim último reduzir o estresse e a sobrecarga emocional, encontrando-se de acordo com o esperado para este tipo de intervençôes (ARRUDA et al., 2008). 
No entanto, um dos grupos se distanciou significativamente do que é apresentado frequentemente na literatura científica sobre o conceito de GAM e promoveu encontros em que se refletissem momentos exclusivamente de recreação, com a manutenção dos laços de amizade e de convívio social. Este resultado evidencia a necessidade de os cuidadores informais socializarem e possuírem outras formas de ocupação e de lazer que sejam legitimadas socialmente (i.e., integrar nestes encontros "destinados a cuidadores" permite, de um ponto de vista social, não ser objeto de críticas por se ausentar da tarefa de cuidador). $\mathrm{O}$ estudo de revisão de Pistrang e colegas (PISTRANG et al., 2008) mostrou que os GAMs e o tipo de suporte que prestam permitem aos cuidadores compensar as deficiências nas redes sociais naturais e criar um contexto comunitário no qual os cuidadores se sentem entendidos (O'CONNOR, 2002) e, em última instância, reconhecidos/valorizados no seu papel. Sendo certo que os GAMs não constituem uma fonte de satisfação de todas as necessidades de interação social, estes devem desenhar alternativas e incentivar a procura de respostas às necessidades dos seus elementos, particularmente nas organizações comunitárias existentes e no núcleo familiar de cada um.

Algumas evidências de estudos de caráter quantiqualitativo sugerem uma associação entre a frequência de GAM e uma melhoria no funcionamento social e familiar, e na perceção de apoio social disponível por parte dos usuários destes serviços (CHIEN et al., 2004; CASTELEIN et al., 2008; COHEN et al., 2012). Nos GAMs analisados neste estudo, o sentimento de atendimento e apoio experimentado pelos seus participantes endossa o valor desta intervenção na comunidade, fazendo eco do preconizado por estudos anteriores sobre os benefícios desta intervenção (GRECO et al., 1999). Enquanto "respostas de continuidade", apresentam-se como recursos valiosos, pois oferecem a possibilidade de os participantes disporem de uma atenção complementar à recebida no âmbito de uma intervenção psicoeducativa, que pode ajudar o cuidador a superar a situação de isolamento que acompanha frequentemente a sua função e garantir uma informação mais útil, dada por pares, para necessidades específicas da vida cotidiana (NAVARRO, 2005). Os GAMs podem, em complementaridade, solidificar redes de vizinhança capazes de garantir apoios pontuais na gestão dos cuidados à pessoa dependente (fato constatado na totalidade dos grupos analisados), bem como estreitar relaçôes com os profissionais de saúde e/ou da 
área social sensíveis às necessidades do cuidador per se, e não do receptor de cuidados, como é mais comum nos serviços de apoio à população mais velha. A este propósito, convém recordar que, apesar de distintos, os locais de realização dos GAMs neste estudo são sempre próximos da população abrangida, sendo locais de referência e de fácil acesso em termos de transporte, onde os cuidadores podem encontrar facilmente o técnico responsável pela dinamização do GAM e se sentirem seguros e confortáveis para partilhar as suas experiências.

De salientar no âmbito dos resultados obtidos, é também a heterogeneidade apresentada pelos membros de cada GAM, a qual parece moldar de modo significativo o funcionamento dos grupos, seja pelas temáticas abordadas (mais ou menos assentes em conteúdos científicos), seja pelas redes de entreajuda propiciadas (distintas entre homens e mulheres), ou pela natureza das atividades recreativas propostas (variáveis consoante a proximidade geográfica dos participantes). Com efeito, o grau de envolvimento dos cuidadores varia em cada grupo em função destas características, às quais acrescem o estatuto socioeconômico, a facilidade de acesso aos locais de realização dos GAMs e, muito provavelmente, o nível de comprometimento com o GAM, nomeadamente expectativas e motivaçôes, as necessidades afetivas e o grau de sobrecarga emocional. Apesar de não ser possível apurar neste estudo estas dimensões para cada um dos participantes, nem tampouco analisar os benefícios individuais em termos de recurso a serviços ou em termos de saúde mental pela natureza descritiva e exploratória da abordagem realizada, importa evidenciar que a investigação tem demonstrado uma associação entre a frequência de um GAM e a redução da taxa de utilização de instalações de internamento (BURTI et al., 2005), bem como uma utilização mais ajustada de serviços de saúde (CHIEN et al., 2007). A literatura aponta, ainda, para o ajustamento psicológico positivo do cuidador (CHIEN et al., 2007; CHIEN et al., 2008; FALCÃO; BUCHERMALUSCHKE, 2009) e a diminuição da sobrecarga e melhoria do bem-estar psicológico (CHIEN et al., 2006; CHIEN et al., 2008; CHIEN et al., 2011; COHEN et al., 2012), com redução dos níveis de preocupação, depressão major e sentimento de incapacidade (LUCKSTED et al., 2008). Todos estes benefícios serão importantes focos de análise numa investigação longitudinal dos cuidadores que integram os GAMs aqui considerados. 


\section{Conclusão}

Em Portugal, os GAMs orientados para os cuidadores de pessoas com demência são ainda escassos, embora, na generalidade, sejam um movimento social contemporâneo significativo e em expansão. Os últimos anos marcam o surgimento de iniciativas comunitárias de entreajuda e suporte ativo entre subpopulações mais vulneráveis que partilham problemas comuns, contando com a colaboração de técnicos, familiares e da comunidade em geral. O Projeto CQC evidenciou a pertinência e a necessidade de uma abordagem mista na conceção de medidas comunitárias de apoio aos cuidadores, garantindo o seu suporte no final dos Programas Psicoeducativos sob a forma de GAM.

A literatura científica sobre as medidas de apoio aos cuidadores tem mostrado que a combinação de diferentes formatos, áreas de intervenção e procedimentos possibilita uma atuação integrada e complementar no apoio prestado aos cuidadores informais (CHIEN et al., 2011). É, pois, fundamental, nesse âmbito, compreender os mecanismos de funcionamento dos GAMs e os seus efeitos psicossociais e comunitários e conscientizar decisores políticos e direçōes das organizaçôes públicas de assistência para a importância desta tipologia de intervenção considerada de baixo custo. No entanto, mais estudos são necessários para a avaliação dos benefícios dos GAMs nos domínios clínico, social, funcional e econômico, comparativamente a outros meios interventivos (e.g., grupos psicoeducativos e serviços individuais standard), através de estudos longitudinais quantiqualitativos que considerem medidas objetivas e subjetivas (CHIEN, 2008). Futuras pesquisas devem também determinar que medida e formato/ configuração deste tipo de intervenção (e.g., se monitorizada por um técnico ou de gestão autônoma por parte dos cuidadores; se alocada a um contexto de saúde específico, como o Centro de Saúde) influencia os benefícios obtidos pelos cuidadores abrangidos de forma a ajudar as famílias a melhorar o seu nível de funcionamento para lidar com as exigências relativas ao processo de cuidar. ${ }^{2}$

\section{Referências}

ALZHEIMER DISEASE INTERNATIONAL. Starting a Self-Help Group. London: Alzheimer's Disease International, 2000.

ARRUDA, M.C.; ALVAREZ, A.M.; GONÇALVES, L.H.T. O Familiar Cuidador de Portador de Doença de Alzheimer Participante de Um grupo de Ajuda Mútua. Cienc Cuid Saude, v. 7, n. 3, p. 339-345, 2008. 
BRODATY, H.; GREEN, A.; KOSCHERA, A. Meta-analysis of psychosocial interventions for caregivers of people with dementia. J Am Geriatr Soc, v. 51, n. 5, p. 657-664, 2003.

BURTI, L.; AMADDEO, F.; AMBROSI, M. et al. Does additional care provided by a consumer self-help group improve psychiatric outcome? A study in an Italian communitybased psychiatric service. Community Ment Health J, v. 41, n. 6, p. 705-720, 2005.

CASTELEIN, S.; BRUGGEMAN, R.; VAN BUSSCHBACH, J. et al. The effectiveness of peer support groups in psychosis: a randomized controlled trial. Acta Psychiatr Scand, v. 118, n. 1, p. 64-72, 2008.

CHIEN, L.Y.; CHU, H.; GUO, J.L. et al. Caregiver support groups in patients with dementia: a meta-analysis. Int J Geriatr Psychiatry, v. 26, n. 10, p. 1089-1098, 2011.

CHIEN, W.T. Effectiveness of Psychoeducation and Mutual Support Group Program for Family Caregivers of Chinese People with Schizophrenia. Open Nurs J, v. 2, n. 1, p. 28-39, 2008.

CHIEN, W.T.; CHAN, S.W.C.; MONISSEY, J. Effectiveness of mutual support and psychoeducation group interventions for family caregivers of patients with schizophrenia. Hong Kong Med J, v. 13, n. Suppl 4, p. S17-20, 2007.

CHIEN, W.T.; CHAN, S.W.C.; THOMPSON, D.R. Effects of a mutual support group for families of Chinese people with schizophrenia: 18-month follow-up. Br J Psychiatry, v. 189, n. 1, p. 41-49, 2006.

CHIEN, W.T.; NORMAN, I.; THOMPSON, D.R. A randomized controlled trial of a mutual support group for family caregivers of patients with schizophrenia. Int J Nurs Stud, v. 41, n. 6, p. 637-49, 2004.

CHIEN, W.T.; THOMPSON, D.R., NORMAN, I. Evaluation of a peer-led mutual support group for Chinese families of people with schizophrenia. Am J Community Psychol, v. 42, n. 1-2, p. 122-34, 2008.

COHEN, A.; RAJA, S.; UNDERHILL, C. et al. Sitting with others: mental health selfhelp groups in northern Ghana. Int J Ment Health Syst, v. 6, n. 1, p. 1, 2012.

DUCHARME, F.; KERGOAT, M.J.; COULOMBE, R. et al. Unmet support needs of early-onset dementia family caregivers: a mixed-design study. BMC Nursing, v. 13, n. 1, p. $1-10,2014$.

FALCÃO, D.V.S.; BUCHER-MALUSCHKE, J.S.N.F. Cuidar de Familiares Idosos com Doença de Alzheimer: uma reflexão sobre aspectos psicossociais. Psicologia em Estudo, v. 14, n. 4, p. 777-786, 2009.

FLICK, U. Métodos Qualitativos na Investigação Cientifica. Lisboa: Monitor, 2005.

FINN, L.D.; BISHOP, B.J.; SPARROW, N. Capturing Dynamic Processes of Change in GROW Mutual Help: Groups for Mental Health. Am J Community Psychol, v. 44, n. 1, p. 302-315, 2009. 
GÓMEZ GÓMEZ, M.M. Estar ahí, al cuidado de un paciente con demencia. Invest Educ Enferm, v. 25, n. 2, p. 60-71, 2007.

GONÇALVES-PEREIRA, M.; SAMPAIO, D. Psicoeducação familiar na demência: da clínica à saúde publica. Revista Portuguesa de Saúde Publica, v. 29, n. 1, p. 3-5, 2011.

GRECO, M.G.; CALENTI, J.C.M.; ZAMORANO, B.G. et al. Evaluación y resultados de la instauración de un programa de terapia de grupo dirigido a un colectivo de cuidadores familiares de enfermos de Alzheimer. Interv Psicosoc, v. 8, n. 1, p. 109-116, 1999.

GUERRA, S.R.; DEMAIN, S.; FIGUEIREDO, D.; SOUSA, L. Post-Intervention referral service (PIRS): Supporting families of people with dementia after a psychoeductional program. Activ Adapt Aging, v. 37, n. 4, p. 319-334, 2013.

INSTITUTO NACIONAL DE ESTATÍSTICA (INE). Anuário Estatístico de Portugal, Lisboa: INE, 2008.

JEREZ, C.H.; LÓPEZ, M.C. Caracterización de los grupos de ayuda mutua para cuidadores de familiares enfermos de alzheimer: un análisis exploratorio. Rev Esp Geriatr Gerontol, v. 43, n. 5, p. 308-315, 2008.

LUCKSTED, A., STEWART, B., FORBES, C.B. Benefits and Changes for Family to Family Graduates. Am J Community Psychol, v. 42, n. 1-2, p. 154-66, 2008.

MAIA, H.; VASQUES, M.; VIEIRA, J. et al. Manual de Ajuda Mútua. Cadernos SNR. Lisboa: Secretariado Nacional para a Reabilitação e Integração das Pessoas com Deficiência, 2002.

NAVARRO, J.R. Asociaciones y Grupos de Ayuda Mutua: un nuevo paradigma en el ámbito de la salud. Revista Internacional de Ciencias Sociales y Humanidades, SOCIOTAM, v. 14, n. 1, p. 83-99, 2004.

. Un análisis de los grupos de ayuda mutua y el movimiento asociativo en el ámbito de la salud: adicciones y enfermedades crónicas. Revista de Antroplogía Experimental, v. 5, n. 1, Texto 13, 2005.

O'CONNOR, D.L. Toward empowerment: ReVisioning family support groups. Soc Work Groups, v. 25, n. 4, p. 37-56, 2002.

PISTRANG, N.C.; BARKER, C.; HUMPHREYS, K. Mutual Help Groups for Mental Health Problems: A Review of Effectiveness Studies. Am J Community Psychol, v. 42, n. 1-2, p. 110-121, 2008.

RAPPAPORT, J. Narrative studies, personal stories, and identity. Transformation in the mutual help context. J Appl Behav Sci, v. 29, n. 2, p. 239-256, 1993.

RIBEIRO, O.; PIRES, C.L.; BRANDÃO, D. et al. Cuidar de Quem Cuida - Projecto de Intervenção Municipal na Regiāo EDV. Relatório Final. Porto: UNIFAI/ICBAS-UP, 2013.

SORIANO, F.R. Los “desconocidos” grupos de ayuda mutual. Cuadernos de Trabajo Social, v. 11, n. 1, p. 251-263, 1998. 
${ }^{1}$ A Junta de Freguesia, no contexto português, é o órgão executivo colegial de uma área geográfica (designada de freguesia), que dispóe constitucionalmente de poder e meios para prossecução de interesses próprios da população respectiva.

${ }^{2}$ O. Ribeiro elaborou o desenho do estudo, analisou os dados e redigiu o artigo. R. Almeida participou da revisão conceitual, coleta de dados e análise. C. Barbosa, da coleta de dados e da revisão crítica do conteúdo intelectual do artigo. N. Duarte, da análise e interpretação dos dados e da redação do manuscrito. D. Brandão, da coleta de dados e da redação do artigo. 
Support Groups for Informal Caregivers of people with dementia: towards a communitarian helping ethos

Within community interventions that involve entities from both the health and the social sectors, group interventions for informal caregivers of individuals with dementia have received increased attention and are both a contemporary research topic and a subject of professional reflection. This study reports a set of distinct Support Groups for Informal Caregivers that were implemented after the caregivers' participation in a psychoeducational program. Based upon a community intervention project implemented in North Portugal (Caring for the Caregiver Project), the study describes five support groups established in five different municipalities and assumed by local agents from the health and/or social sectors. It focuses on each group's main objectives and functioning characteristics as a continuity response that was organized at a broad community level. Main findings stress the importance of these support groups on maintaining the caregivers' informal social network and mutual support system within a context of reduced services explicitly targeted for this population, and to the need of having a professional who assumes the role of organizing the sessions. Authors conclude on the relevance of support groups as a continuous and lively community intervention targeted at helping family carers of people with dementia.

> Keywords: informal caregivers; dementia; support groups; elderly. 